\title{
Stationary statistical experiments and optimal estimator for predictable component
}

\author{
DMitri Koroliouk
}

(Presented by S. Makhno)

\begin{abstract}
A stationary autoregression process given by a difference stochastic equation is characterized by a two-dimensional covariance matrix under stationarity conditions. The optimal estimator function represented by a square variation of the martingale is used to obtain consistent estimators for the parameter of a predictable component.
\end{abstract}

2010 MSC. 46N30, 60J70, 62F05.

Key words and phrases. Stationary autoregression process, optimal estimator function, consistent statistics, evaluation parameters.

\section{Introduction}

The statistical analysis of real measurements is particularly important, when the observation of physical data (biological, chemical, etc.) has often no justification in the form of a physical theory.

In numerous complex systems like biological processes with equilibrium, the dynamics of the concentration or frequencies of the presence of some predefined attribute can be described by a mathematical model of binary statistical experiments. The model is based on the validation of elementary hypotheses about the presence or absence of the predefined attribute in the set of elements that make up a complex system [1].

It is assumed that:

1. all the elements that make up the system can gain or lose the said attribute in the course of time; i.e, the relative frequency of an attribute is a dynamic variable;

2. the basic objects of our study are statistical experiments (SEs) characterized by relative frequencies of the presence of an attribute in a sample of fixed volume at every time instant;

Received 17.10.2015 
3. the average result of a next experiment depends on the average result of the present experiment. Such a relationship is called the persistent regression and used as a fundamental condition for the subsequent analysis of the model.

The method of construction and exploration of the proposed mathematical model is based on the analysis of the following basic properties [2] of statistical experiments:

1. Persistent regression.

2. Equilibrium value and fluctuations, as well as their asymptotic behavior.

3. Approximation by the normal process of autoregression.

The present work introduces a new computational method and the corresponding statistical estimators of parameters characterizing the transitional mobility of the dynamical frequency (concentration).

The model is based on the stationarity conditions for statistical experiments given by a difference stochastic equation with predictable linear component and with additional stochastic component determined by a stationary, in wide meaning, random sequence.

The martingale approach using the martingale-differences in the analysis of autoregressive models is described in books [3,4]. In particular, the conditional least square method based on martingale-differences is considered, for example, in works [5-7]. In [8, Ch. 14], Liptser and Shiryaev considered the statistical problems for stationary random sequences with fractionally rational spectrum, by using the filtering equation.

The aim of this article is to apply the martingale approach proposed by Heyde in [3] to the construction of an optimal estimator of the predictable component parameter by involving the square variation of the martingale component.

\section{Statement of the problem}

Definition 1. A statistical experiment with persistent regression [1,2] is given by the solution of the difference stochastic equation

$$
\Delta \alpha_{t}=-V_{0} \alpha_{t}+\Delta \mu_{t}, \quad \Delta \alpha_{t}:=\alpha_{t+1}-\alpha_{t}, \quad t \in \mathcal{N}_{0}=0,1,2,3, \ldots
$$

The stochastic component $\Delta \mu_{t}, t \in \mathcal{N}_{0}$, is given by the martingaledifferences

$$
\Delta \mu_{t}:=\mu_{t+1}-\mu_{t}, \quad t \in \mathcal{N}_{0}=0, \quad \mu_{0}=0,
$$

generated by a stationary square integrable martingale $\mu_{t}, t \in \mathcal{N}_{0}$, which is characterized by two first moments:

$$
E\left(\Delta \mu_{t}\right)=0, \quad E\left(\Delta \mu_{t}\right)^{2}=\sigma^{2}, \quad \forall t \in \mathcal{N}_{0} .
$$


The initial value $\alpha_{0}$ in (1)-(2) is a random variable independent of $\Delta \mu_{t}, t \geq 1$. The predictable parameter $V_{0}$ satisfies the following limitation:

$$
0<V_{0}<2
$$

\section{Stationary statistical experiments}

A stationary, in a wide meaning, process with discrete time is determined by the covariance function [9, Ch. VII].

In our model with (1)-(3), the stationarity in a wide meaning is ensured by the condition

$$
\operatorname{cov}\left(\alpha_{t}, \alpha_{t}\right)=E \alpha_{t}^{2}=\sigma_{0}^{2}, \quad t \in \mathcal{N}_{0} .
$$

In this connection, it is necessary to establish conditions for the initial distribution which assure the stationarity of Eqs. (1)-(3) in a wide meaning.

Theorem 1. The stochastic equation (1)-(3) presents a stationary random sequence in a wide meaning iff the following relations take place:

$$
E \alpha_{0}=0, \quad E \alpha_{0}^{2}=\sigma_{0}^{2}=\sigma^{2} / V_{0}\left(2-V_{0}\right) .
$$

Proof. The statement of Theorem 1 is carried out by induction.

Let $B_{t}:=E \alpha_{t}^{2}=\sigma_{0}^{2}, t \leq T$. The formula (1) implies that

$$
E\left[\Delta \alpha_{t}\right]^{2}=E\left[-V_{0} \alpha_{t}+\Delta \mu_{t}\right]^{2}=V_{0}^{2} \sigma_{0}^{2}+\sigma^{2} .
$$

By the relation $\Delta \alpha_{t}:=\alpha_{t+1}-\alpha_{t}$, dispersion (6) can be represented as follows:

$$
E\left[\Delta \alpha_{t}\right]^{2}=B_{t+1}+\left(2 V_{0}-1\right) \sigma_{0}^{2} .
$$

In other words, taking together (6) and (7), we have

$$
B_{t+1}-B_{t}=\left(V_{0}^{2}-2 V_{0}\right) \sigma_{0}^{2}+\sigma^{2}=0 .
$$

Hence, for all $t \in \mathcal{N}_{0}, B_{t}=\sigma_{0}^{2}$.

The necessity of conditions (5) is obvious.

Remark 1. The limitation condition (3) provides the correctness of the statistical stationarity condition (5). 


\section{Statistics for stationary statistical experiments}

The covariation analysis can be used for developing the statistics for the stationary statistical experiments (1)-(5).

Theorem 2. The stationary two-component vectors $\left(\alpha_{t}, \Delta \alpha_{t}\right), t \in \mathcal{N}_{0}$ (1)-(5) can be described by the following covariation functions:

$$
\begin{gathered}
R(s):=E\left[\alpha_{t} \alpha_{t+s}\right]=\sigma_{0}^{2} q^{s}, R^{0}(s):=E\left[\alpha_{t} \Delta \alpha_{t+s}\right]=-V_{0} \sigma_{0}^{2} q^{s}, s \geq 0, \\
R^{\Delta}(s):=E\left[\Delta \alpha_{t} \Delta \alpha_{t+s}\right]=-V_{0} \sigma_{0}^{2} q^{s-1}, s \geq 1, q:=1-V_{0} .
\end{gathered}
$$

The covariations are calculated as follows:

$$
R:=R(0)=\sigma_{0}^{2}, R^{0}:=R^{0}(0)=-V_{0} \sigma_{0}^{2}, R^{\Delta}=R^{\Delta}(0):=2 V_{0} \sigma_{0}^{2} .
$$

Proof. The first and third covariations in (9) are obvious.

The second covariation in (9) is calculated, by using Eq. (1) and relation (6).

The covariation functions (8) for the two-component vectors $\left(\alpha_{t}, \Delta \alpha_{t}\right)$, $t \in \mathcal{N}_{0}$ are proved by induction.

Remark 2. Covariations (9) generate, as estimators, the following statistics:

$$
R_{T}:=\frac{1}{T} \sum_{t=0}^{T-1} \alpha_{t}^{2}, R_{T}^{\Delta}:=\frac{1}{T} \sum_{t=0}^{T-1}\left(\Delta \alpha_{t}\right)^{2}, R_{T}^{0}:=\frac{1}{T} \sum_{t=0}^{T-1} \alpha_{t} \Delta \alpha_{t} .
$$

In what follows, we use the following proposition.

Presupposition 1. Estimators (10) are consistent in the meansquare meaning:

$$
E\left[R_{T}-R\right]^{2} \rightarrow 0, E\left[R_{T}^{\Delta}-R^{\Delta}\right]^{2} \rightarrow 0, E\left[R_{T}^{0}-R^{0}\right]^{2} \rightarrow 0, T \rightarrow \infty .
$$

Remark 3. The consistency of estimators (11) for Gaussian SE follows from the condition [9, Ch. VII. Annex. and exerc., ex. 23]:

$$
\frac{1}{T} \sum_{s=0}^{T-1} R^{2}(s) \stackrel{L^{2}}{\rightarrow} 0, \quad T \rightarrow \infty .
$$

Remark 4. The consistency of estimators (11) also has place for the bounded SE.

Proposition 1. Statistics (10) generates the following two estimators of the predictable component parameter $V_{0}$ :

$$
V_{T}^{0}=-R_{T}^{0} / R_{T}, \quad V_{T}^{\Delta}=R_{T}^{\Delta} / 2 R_{T},
$$

which, as will be shown below, are both consistent in mean square and asymptotically unbiased. 


\section{Martingale approach}

The problem of optimal estimators for the predictable parameter $V_{0}$ can be solved by the quasilikelihood approach represented in monograph [3, Ch. 2]. The initial statistics for the stationary SE (1)-(5) are given by the trajectory

$$
\dot{G}_{T}(V):=\left(\alpha_{t}, 0 \leq t \leq T\right),
$$

and the martingale-differences

$$
G_{T}(V):=\left(\Delta \mu_{t}, 0 \leq t \leq T\right),
$$

or, in the equivalent form,

$$
G_{T}(V):=\left(\Delta \alpha_{t}+V \alpha_{t}, 0 \leq t \leq T\right) .
$$

In (15)-(16), the predictable parameter $V$ is unknown and satisfies the condition $0<V<2$. Following the idea of C. Heyde, we introduce quasiscore functional (QSF).

Definition 2. The normalized $Q S F$ is defined by the covariation ${ }^{1}$

$$
\mathcal{E}(V):=E\left(G_{T}(V), G_{T}(V)\right) / E\left(\dot{G}_{T}(V), \dot{G}_{T}(V)\right) .
$$

Proposition 2. The covariation function in (17) gives the following result:

$$
\mathcal{E}(V)=V^{2}-2 V V_{0}+2 V_{0} .
$$

The martingale statistics (14)-(15) and trajectories (16) generate the following covariation functions in (17):

$$
\begin{aligned}
E\left(G_{T}(V), G_{T}(V)\right) & =E \sum_{t=1}^{T} \Delta \mu_{t}^{2}=E \sum_{t=1}^{T}\left(\Delta \alpha_{t}+V \alpha_{t}\right)^{2} \\
& =\left[V^{2}-2 V V_{0}+2 V_{0}\right] \cdot T \sigma_{0}^{2}
\end{aligned}
$$

and

$$
E\left(\dot{G}_{T}(V), \dot{G}_{T}(V)\right)=E \sum_{t=1}^{T} \alpha_{t}^{2}=T \sigma_{0}^{2},
$$

where $V_{0}$ is a true value of the parameter $V$.

So, the normalized quasiscore function does not depend on the time parameter $T$.

\footnotetext{
${ }^{1}(\cdot, \cdot)$ is a scalar product of two vectors
} 
Corollary 1. Function (18) is positive definite and admits the following representation:

$$
\mathcal{E}(V)=\left(V-V_{0}\right)^{2}+\mathcal{E}^{0}, \quad \mathcal{E}^{0}:=\sigma^{2} / \sigma_{0}^{2}=V_{0}\left(2-V_{0}\right) .
$$

The parameter $\mathcal{E}^{0}$ is called the stationarity coefficient and plays an important role in the optimal estimation.

\section{Optimal estimator function (OEF)}

Following C. Heyde [3], we introduce the normalized estimator function.

Definition 3. The optimal estimator function is given by the relation

$$
\widehat{\mathcal{E}}_{T}(V):=\mathcal{E}_{T}(V) / R_{T},
$$

where, by definition,

$$
\mathcal{E}_{T}(V):=\frac{1}{T} \sum_{t=1}^{T}\left[\Delta \alpha_{t}+V \alpha_{t}\right]^{2}
$$

which is a square variation of the martingale

$$
\mu_{t}:=\sum_{k=0}^{t-1} \Delta \mu_{k}, \quad t \geq 1, \quad \mu_{0}=0 .
$$

The normalizing statistics $R_{T}$ is defined in (10).

Theorem 3. The optimal estimator statistics $V_{T}^{0}$ for the predictable parameter $V_{0}$ is derived from the optimality relation:

$$
\min _{0<V<2} \mathcal{E}_{T}(V)=\mathcal{E}_{T}\left(V_{T}^{0}\right)=\mathcal{E}_{T}^{0}, \quad \mathcal{E}_{T}^{0}:=V_{T}^{\Delta}-\left(V_{T}^{0}\right)^{2},
$$

and has the following explicit form:

$$
V_{T}^{0}=-\sum_{t=1}^{T} \alpha_{t} \Delta \alpha_{t} / \sum_{t=1}^{T} \alpha_{t}^{2}=-R_{T}^{0} / R_{T}
$$

where the statistics $V_{T}^{0}$ and $V_{T}^{\Delta}$ are defined in (13). 
Proof. The calculation of the OEF by formula (23) gives the following result:

$$
\mathcal{E}_{T}(V)=V^{2} R_{T}+2 V R_{T}^{0}+R_{T}^{\Delta} .
$$

Now, the optimal estimator function (22) can be obtained by the division of (27) by the normalized statistics defined in (10):

$$
\widehat{\mathcal{E}}_{T}(V):=V^{2}-2 V V_{T}^{0}+V_{T}^{\Delta}=\left(V-V_{T}^{0}\right)^{2}+V_{T}^{\Delta}-\left(V_{T}^{0}\right)^{2},
$$

where the statistics $V_{T}^{0}$ and $V_{T}^{\Delta}$ are defined in (13).

The last relation provides the statement of Theorem 3 .

Remark 5. It is easy to verify the following convergence:

$$
V_{T}^{\Delta} \stackrel{P}{\rightarrow} 2 V_{0}, \quad V_{T}^{0} \stackrel{P}{\rightarrow}-V_{0}, \quad T \rightarrow \infty .
$$

So, the residual term of the optimal estimator function in (25) represents the estimator for the stationary coefficient $\mathcal{E}^{0}:=\sigma^{2} / \sigma_{0}^{2}$ :

$$
\mathcal{E}_{T}^{0} \stackrel{P}{\rightarrow} V_{0}\left(2-V_{0}\right)=\sigma^{2} / \sigma_{0}^{2}, \quad T \rightarrow \infty .
$$

\section{Gaussian stationary statistical experiment}

In a particular case, the Gaussian stationary statistical experiment is given by a solution of the following difference stochastic equation:

$$
\Delta \alpha_{t}=-V_{0} \alpha_{t}+\Delta W_{t}, \quad t \in \mathcal{N}_{0} .
$$

The stochastic component $\Delta W_{t}$ is given by a martingale-difference of the normal sequence $W_{t}, t \in \mathcal{N}_{0}[10$, Ch. 2 , p. 6]:

$$
\Delta W_{t}:=W_{t+1}-W_{t}, \quad t \in \mathcal{N}_{0}, \quad W_{0}=0,
$$

which is characterized by two first moments:

$$
E\left(\Delta W_{t}\right)=0, \quad E\left(\Delta W_{t}\right)^{2}=\sigma^{2}, \quad t \in \mathcal{N}_{0} .
$$

The initial value of SE (1)-(2) is a normally distributed random variable independent of $\Delta W_{t}, t \geq 1$. The predictable parameter $V_{0}$ satisfies limitation (3).

The problem of optimal estimator for the predictable parameter $V_{0}$ can be solved now, by using the likelihood function for a stationary Gaussian sequence $W_{t}, t \in \mathcal{N}_{0}$, which admits the following representation:

$$
\ln L\left(V, W_{T}\right)=-\frac{1}{2 \sigma^{2}} \sum_{t=0}^{T-1}\left(\Delta W_{t}\right)^{2}+P_{0 T},
$$


where

$$
W_{T}:=\frac{1}{T} \sum_{t=0}^{T-1}\left(\Delta W_{t}\right)^{2},
$$

and $P_{0 T}$ is a constant which does not depend on $V$.

Now, the normalized likelihood estimating function for the predictable parameter $V_{0}$ can be represented, by using the relation (see (33)-(34) and $(22)-(27))$

$$
\ln L\left(V, W_{T}\right)=-\frac{R_{T}}{2 \sigma^{2}} \widehat{\mathcal{E}}_{T}(V)+P_{0},
$$

in the form

$$
\begin{gathered}
\widehat{\mathcal{E}}_{T}(V):=\frac{1}{T} \sum_{t=0}^{T-1}\left(\Delta W_{t}\right)^{2} / R_{T}=V^{2}-2 V V_{T}^{0}+V_{T}^{\Delta}= \\
=\left(V-V_{T}^{0}\right)^{2}+V_{T}^{\Delta}-\left(V_{T}^{0}\right)^{2} .
\end{gathered}
$$

By Theorem 3, the optimal estimator statistics $V_{T}^{0}$ for the predictable parameter $V_{0}$ is given by formula $(26)$.

Corollary 2. The optimal estimator statistics $V_{T}^{0}$, reported in Theorem 3 for the normalized estimator function (36) coincides with the maximum likelihood estimation expressed by the logarithm of the likelihood function (33).

\section{Multivariate stationary statistical experiments}

The martingal approach to the optimal estimation of the predictable parameter $V_{0}$ realized in Sections 5-6 for the stationary statistical experiments (1)-(5) in the one-dimensional case can be extended to multivariate statistical experiments.

Now, the multivariate model of stationary statistical experiments is given by a solution of the difference stochastic equation

$$
\Delta \alpha_{t}=-\mathbb{V}_{0} \alpha_{t}+\Delta \mu_{t}, \quad t \geq 0
$$

The predictable component is determined by the regression matrix

$$
\mathbb{V}:=\left[V_{k m} ; 1 \leq k, m \leq M\right]
$$

which satisfies the additional stationarity condition

the matrix $\mathbb{Q}:=\mathbb{I}-\mathbb{V}$ has the eigenvalues strictly inside a unit circle. 
The stochastic component is defined by the square integrable stationary martingale $\mu_{t}=\left(\mu_{t}^{k}, 1 \leq k \leq M\right), t \in \mathcal{N}_{0}$, which is characterized by two first moments

$$
E \Delta \mu_{t}=0, \quad E\left[\Delta \mu_{t} \Delta \mu_{t}^{\prime}\right]=\mathbb{B}, \quad t \geq 0 .
$$

Here, the martingale-differences are defined as follows:

$$
\Delta \mu_{t}:=\mu_{t+1}-\mu_{t}, \quad t \in \mathbb{N}_{0}, \quad \mu_{0}:=0 .
$$

Under condition (S) by analogy with Theorem 1, the statistical stationarity for the statistical experiments (37)-(38) supposes that the initial value $\alpha_{0}$ is a random variable independent of $\Delta \mu_{t}, t \geq 1$, and satisfies the following condition (cf. (5)):

$$
E \alpha_{0}=0, \quad E\left[\alpha_{0} \alpha_{0}^{\prime}\right]=\mathbb{R} .
$$

The positive definite matrix $\mathbb{R}$ is determined by a solution of the following matrix stationarity equation:

$$
\mathbb{B}=\mathbb{V}_{0} \mathbb{R}+\mathbb{R} \mathbb{V}_{0}^{\prime}-\mathbb{V}_{0} \mathbb{R} \mathbb{V}_{0}^{\prime} .
$$

In other words, the stationary SE (37)-(38) is a stationary process in a wide meaning:

$$
E \alpha_{t}=0, E\left[\alpha_{t} \alpha_{t}^{\prime}\right]=\mathbb{R}, E\left[\alpha_{t+s} \alpha_{t}^{\prime}\right]=\mathbb{R}(s)=(\mathbb{I}-\mathbb{V})^{s} \mathbb{R} \forall t \in \mathcal{N}_{0} .
$$

By analogy with Theorem 2 below, the stationary SE defined by (37)(40) is completely determined by the following covariation matrices:

$$
\begin{gathered}
E\left[\alpha_{t} \alpha_{t}^{\prime}\right]=R, \quad E\left[\Delta \alpha_{t} \Delta \alpha_{t}^{\prime}\right]=R^{\Delta}, \\
E\left[\Delta \alpha_{t} \alpha_{t}^{\prime}\right]=R^{0}, \quad E\left[\alpha_{t} \Delta \alpha_{t}^{\prime}\right]=R^{0^{\prime}} .
\end{gathered}
$$

Following [3], the martingale approach in the statistical analysis for the stationary SE (37)-(41) involves a square variation of martingale (38):

$$
\begin{aligned}
\mathcal{E}_{T}(\mathbb{V}) & :=\frac{1}{T} \sum_{t=0}^{T-1} \Delta \mu_{t} \Delta \mu_{t}^{\prime}=\frac{1}{T} \sum_{t=1}^{T}\left(\Delta \alpha_{t}+\mathbb{V} \alpha_{t}\right)\left(\Delta \alpha_{t}+\mathbb{V} \alpha_{t}\right)^{\prime} \\
& =\mathbb{V} \mathbb{R}_{T} \mathbb{V}^{\prime}+\mathbb{V} \mathbb{R}_{T}^{0^{\prime}}+\mathbb{R}_{T}^{0} \mathbb{V}^{\prime}+\mathbb{R}_{T}^{\Delta},
\end{aligned}
$$

where the following matrix statistics are used:

$$
\begin{aligned}
& \mathbb{R}_{T}:=\frac{1}{T} \sum_{t=1}^{T} \alpha_{t} \alpha_{t}^{\prime}, \quad \mathbb{R}_{T}^{0}:=\frac{1}{T} \sum_{t=1}^{T} \Delta \alpha_{t} \alpha_{t}^{\prime}, \\
& \mathbb{R}_{T}^{0^{\prime}}:=\frac{1}{T} \sum_{t=1}^{T} \alpha_{t} \Delta \alpha_{t}^{\prime}, \quad \mathbb{R}_{T}^{\Delta}:=\frac{1}{T} \sum_{t=1}^{T} \Delta \alpha_{t} \Delta \alpha_{t}^{\prime} .
\end{aligned}
$$


Proposition 3. The optimal estimator function given by Definition 3 has the representation

$$
\mathcal{E}_{T}(\mathbb{V})=\mathcal{E}_{T}^{0}\left(\mathbb{V}, \mathbb{V}_{T}^{*}\right)+\mathcal{B}_{T},
$$

where the following positive definite matrix is used:

$$
\mathcal{E}_{T}^{0}\left(\mathbb{V}, \mathbb{V}_{T}^{*}\right)=\left(\mathbb{V}-\mathbb{V}_{T}^{*}\right) \mathbb{R}_{T}\left(\mathbb{V}-\mathbb{V}_{T}^{*}\right)^{\prime} .
$$

The statistics $V_{T}^{*}$ has the following form:

$$
\mathbb{V}_{T}^{*}=-\mathbb{R}_{T}^{0} \mathbb{R}_{T}^{-1} .
$$

The residual term has the representation

$$
\mathcal{B}_{T}=\mathbb{R}_{T}^{\Delta}-\mathbb{R}_{T}^{0} \mathbb{R}_{T}^{-1} \mathbb{R}_{T}^{0^{\prime}}
$$

or, in another form,

$$
\mathcal{B}_{T}=\mathbb{V}_{T}^{*} \mathbb{R}_{T}+\mathbb{R}_{T} \mathbb{V}_{T}^{* \prime}-\mathbb{V}_{T}^{*} \mathbb{R}_{T} \mathbb{V}_{T}^{* \prime} .
$$

It is not difficult to deduce the following relation:

$$
\mathcal{E}_{T}(\mathbb{V})-\mathcal{E}_{T}^{0}\left(\mathbb{V}, \mathbb{V}_{T}^{*}\right)=\mathcal{B}_{T},
$$

which yields (44).

Theorem 4. The estimating statistics $\mathbb{V}_{T}^{*}$ for the predictable matrix $\mathbb{V}_{0}$ is derived from the optimality relation

$$
\min _{V} \mathcal{E}_{T}(\mathbb{V})=\min _{V}\left[\mathcal{E}_{T}^{0}\left(\mathbb{V}, \mathbb{V}_{T}^{*}\right)+\mathcal{B}_{T}\right]=\mathcal{B}_{T},
$$

and the estimating statistics $\mathbb{V}_{T}^{*}$ is given by relation (46).

The minimum of the optimality relation (44) is reached with (48).

Remark 6. It is easy to verify that the optimal statistics (46) is consistent in mean square:

$$
\text { l.i.m. } T \rightarrow \infty \mathbb{V}_{T}^{*}=\mathbb{V}_{0} \text {. }
$$

\section{Gaussian Markovian stationary multivariate SE}

A Gaussian Markovian stationary multivariate SE (GM-SSE) $\alpha_{t}, t \in$ $\mathcal{N}_{0}$ is determined by two moments:

$$
E \alpha_{t}=a, \quad E\left[\alpha_{t} \cdot \alpha_{t}^{\prime}\right]=R, \quad t \geq 1 .
$$

The stationarity condition means that the initial value $\alpha_{0}$ is a Gaussian random variable with

$$
E \alpha_{0}=a, \quad E\left[\alpha_{0} \cdot \alpha_{0}^{\prime}\right]=R .
$$


Theorem 5. The GM-SSE (50)-(51) can be defined by the difference stochastic equation

$$
\Delta \alpha_{t}^{0}=-\mathbb{V} \alpha_{t}^{0}+\boldsymbol{\sigma} \Delta W_{t+1}, \quad t \in \mathcal{N}_{0} .
$$

Here, by definition, $\alpha_{t}^{0}:=\alpha_{t}-a$, and $\boldsymbol{\sigma}^{2}:=B=\sigma \cdot \sigma^{\prime}$.

The matrices $\mathbb{R}, \mathbb{V}$, and $\mathbb{B}$ are connected as follows:

$$
\mathbb{B}=\mathbb{V} \mathbb{R}+\mathbb{R} \mathbb{V}^{\prime}-\mathbb{V} \mathbb{R} \mathbb{V}^{\prime}
$$

and the Gaussian stationary sequence $W_{t}, t \geq 1$ is defined by the Gaussian martingale-differences:

$$
W_{t+1}:=\sum_{k=0}^{t} \Delta W_{t}, \quad E \Delta W_{t}=0, \quad E\left[\Delta W_{t} \Delta W_{t}^{\prime}\right]=\mathbb{I} .
$$

The proof of Theorem 1 follows from the theorem of normal correlation [8, Th. 13.1 and Lem. 13.4].

Acknowledgements. The author thanks Yu. Mishura and Yu. Kozachenko for their useful constructive advices.

\section{References}

[1] D. Koroliouk, V. S. Koroliuk , N. Rosato, Equilibrium Process in Biomedical Data Analysis: the Wright-Fisher Model // Cybernetics and Systems Analysis, 50 (2014), No. 6, 890-897.

[2] D. Koroliouk, Binary statistical experiments with persistent non-linear regression // TIIMS, 91 (2014), 64-73.

[3] C. C. Heyde, Quasi-Likelihood And Its Application. A General Approach to Optimal Parameter Estimation. Springer Series in Statistics, 1997.

[4] P. Hall, C. C. Heyde, Martingale Limit Theory and its Application, Academic Press, New York, 1980.

[5] P. M. Robinson, Statistical inteference for a random coefficient autoregressive model // Scand. J. Statist., 5 (1978), 163-168.

[6] Z. W. Zhao, D. H. Wang, Y. Zhang, Limit theory for random coefficient first-order autoregressive process under martingale difference error sequence // J. Comp. Alppl. Math., 235 (2011), 2515-2522.

[7] X. Chen, H. Cui, Empirical likehood inference for partial linear models under martingale difference sequence // Statist. Probab. Lett. 78 (2008), 2895-2901.

[8] R. S. Liptser, A. N. Shiryaev, Statistics of stochastic processes. M.: Nauka, 1974 (in Russian).

[9] A. Bulinski, A. N. Shiryaev, Theory of Random Processes. M.: Fismatlit, 2003 (in Russian).

[10] A. A. Borovkov, Mathematical statistics. Estimation of parameters and veriffication of hypothesis. M.: Nauka, 1984 (in Russian). 
[11] A. N. Shiryaev, Probability-1. M.: MCNMO, 2004.

\section{CONTACT INFORMATION}

Dmitri Koroliouk Institute of Telecommunications and Global Information Space

Ukrainian Academy of Sciences

E-Mail: dimitri.koroliouk@ukr.net 